\title{
La conflictualité en discours : le recours à l'injure dans les arènes publiques
}

Conflict in Discourse: Resorting to Insults in Public Arenas

\section{Claire Oger}

\section{(2) OpenEdition}

\section{Journals}

Édition électronique

URL : http://journals.openedition.org/aad/1297

DOI : $10.4000 /$ aad. 1297

ISSN : 1565-8961

Éditeur

Université de Tel-Aviv

Référence électronique

Claire Oger, "La conflictualité en discours : le recours à l'injure dans les arènes publiques », Argumentation et Analyse du Discours [En ligne], 8 | 2012, mis en ligne le 15 avril 2012, consulté le 23 septembre 2019. URL : http://journals.openedition.org/aad/1297 ; DOI : 10.4000/aad.1297

Ce document a été généré automatiquement le 23 septembre 2019.

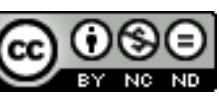

Argumentation \& analyse du discours est mis à disposition selon les termes de la licence Creative Commons Attribution - Pas d'Utilisation Commerciale - Pas de Modification 4.0 International. 


\title{
La conflictualité en discours : le recours à l'injure dans les arènes publiques
}

\author{
Conflict in Discourse: Resorting to Insults in Public Arenas
}

\section{Claire Oger}

1 "Eh bien, finalement, je plaide coupable. J'utilise en effet sans remords les "métaphores zoologiques". Ce qui caractérise la politique [...], c'est qu'il y a des ennemis. Et pourquoi diable, si ce sont de vrais ennemis, me serait-il interdit de les injurier ? $»^{1}$. S'il est rare de revendiquer un droit à l'injure, comme le fait ici Alain Badiou, il l'est moins de reconnaître à la politique son caractère de combat, de lutte parfois sans merci pour l'accès au pouvoir. Dès lors, la disqualification de l'adversaire, y compris dans ses formes brutales, peut être présentée comme une «règle du jeu politique ", à laquelle il serait naiff de vouloir échapper.

2 A l'origine de notre questionnement se situe précisément la question des conditions dans lesquelles l'usage de l'injure - communément réprouvé - peut être présenté comme licite, voire légitime, dans certains contextes polémiques (Oger 2003). Inversement, nous nous sommes intéressée à des discours qui la présentaient comme une forme intolérable de violence verbale : le cas précis qui nous a intéressée est celui des ouvrages de femmes politiques s'attachant à dénoncer la pratique de l'injure sexiste en politique (Oger 2006, 2008a, 2011). Cet article se propose de poursuivre ce questionnement non par une nouvelle étude de corpus mais par un parcours interdisciplinaire parmi des travaux, récents ou plus anciens, qui relèvent des études argumentatives, de la psychanalyse, de la sociologie, de la science politique ou de l'histoire et qui nous semblent susceptibles de contribuer à la construction d'un cadre de réflexion général sur le sujet. 


\section{Le continuum de l'argumentation polémique, entre psychanalyse et matérialités langagières}

3 Un premier axe d'analyse concerne l'intrication entre argumentation et recours à la violence verbale, dont les polémiques médiatisées entre intellectuels fournissent des exemples emblématiques. En effet, une première forme de légitimation repérable réside dans la continuité que l'on peut établir entre des procédés qui, dans le même texte, relèvent pour certains de la réfutation du propos adverse, et pour d'autres, de l'attaque personnelle. La dimension injurieuse d'un énoncé peut donc se trouver minorée - et comme autorisée - par le co-texte (quand il présente les éléments d'une argumentation construite et étayée) et/ou par le contexte éditorial (s'il s'agit par exemple d'une tribune publiée dans un journal dit « de référence » comme Le Monde) ${ }^{2}$.

4 A la différence des interactions verbales, où l'on peut observer des phénomènes de «montée en tension» (Moïse 2006), il nous a semblé délicat de tracer dans les corpus étudiés ${ }^{3}$ une frontière nette entre des procédés qui relèvent d'une argumentation respectueuse de l'adversaire, et ceux qui appartiennent au registre de la violence verbale car le passage de l'un à l'autre n'y apparaît pas comme un saut qualitatif, mais davantage comme une zone trouble ${ }^{4}$ : pour en interroger les ambiguïtés, le recours aux travaux de la psychanalyste Evelyne Larguèche s'est avéré très éclairant car il nous a permis de repérer comment l'injure dite "spécifique ${ }^{5}$ assurait une forme de continuité entre l'argumentation ad hominem ${ }^{6}$ et l'injure " non-spécifique » : car l'injure qui «spécifie son attaque, la particularise [...], qualifie une personne et pas une autre, [...] dépeint au plus près du vérifiable et du vraisemblable » (Larguèche $2011: 363$ ), abritant en cela un «noyau argumentatif».

5 Ces recherches permettent aussi de comprendre la complexité de l'« effet-injure », et la diversité des procédés ou des comportements qualifiés d'injurieux: insinuations, sourires en coin, allusions, rumeurs, brouhahas, ou même silences peuvent être différenciés d'un point de vue formel, mais se rejoignent et se cumulent dans la perception de l'agression (Oger 2006) 7 . Linguistes et sociolinguistes ont décrit les paradoxes des situations de communication où l'adresse injurieuse, signe de complicité, se fait « insulte de solidarité » ou assaut ritualisé de « vannes », mais aussi la façon dont tout mot peut en contexte conflictuel devenir une insulte ${ }^{8}$. Seules les traces de la qualification du procédé par les destinataires, analysées selon une approche métadiscursive, peuvent donc guider l'interprétation, et la mesure de l'« effet-injure » ne peut obéir à une catégorisation a priori.

6 La psychanalyse jette également un éclairage intéressant sur l'ambiguïté des relations entre humour, dérision et agression, soulignée par ailleurs par l'analyse des discours et les sciences de l'information et de la communication (Mercier et al. 2001). Tandis que

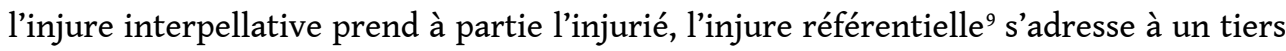
(en présence ou en l'absence de l'injurié) et s'apparente en cela au "mot d'esprit » étudié par Freud (1992 [1905]). Or, en mettant au jour la façon dont le trait d'esprit, décoché pour nuire et pour humilier, traduit une tendance hostile, l'approche proposée par Freud contribue à fragiliser l'excuse humoristique, parfois invoquée par les injurieurs ${ }^{10}$.

7 Enfin, la psychanalyse comme l'analyse du discours des injurié(e)s invitent à considérer avec beaucoup de réserve ou de circonspection la valorisation paradoxale de la violence 
verbale, présentée parfois comme un substitut somme toute positif à la violence physique, à laquelle elle éviterait au moins de recourir ${ }^{11}$. La mise à mort symbolique de l'adversaire comme procédé langagier (Oger 2003), et dans le cas des femmes, l'humiliation traumatique comparée à celle du viol (Oger 2006) constituent des indices d'une relation - symbolique, à défaut de consécutive - entre violence verbale et violence physique.

8 En désignant des ambiguïtés, et en récusant l'innocuité supposée de pratiques dont elle éclaire les soubassements hostiles, la psychanalyse apporte une contribution qui nous semble décisive à l'analyse des énoncés injurieux et plus généralement du discours polémique. En pointant des lieux de recouvrement entre argumentation et injure, elle interroge également la pertinence de l'antique division entre dialectique et éristique, ou entre dialectique consensuelle et dialectique éristique, décrite par Angenot comme une opposition entre un art de la discussion sereine et un art de la controverse et de la dispute - technique de la "guerre argumentative » où il s'agit de gagner «par tous les moyens », « à tout prix » $(2008: 49,52)$.

\section{Les études argumentatives et la construction des normes}

Dans les corpus que nous avons étudiés, beaucoup de locuteurs tendent pourtant à établir une frontière normative entre pratiques discursives licites et illicites, et à fixer des règles évaluant les comportements de leurs adversaires. Ainsi, nombre de femmes politiques qui dénoncent l'injure sexiste attribuent à leurs injurieurs un ethos guerrier, disqualifié comme une pratique de "meute", et fondé sur un entre-soi machiste et vulgaire. La plupart d'entre elles y opposent l'argument du genre selon lequel les femmes contribueraient à introduire en politique des mœurs plus pacifiques et une forme de modération ${ }^{12}$. Dans ce type de discours, on voit se dessiner des normes implicites ou explicites qui dessinent des conceptions de la parole politique et s'organisent tout particulièrement autour de la place que peut y occuper la conflictualité $^{13}$. Il ne s'agit pas ici de sacrifier à une approche "normative" de l'argumentation ${ }^{14}$, mais bien de s'intéresser à la construction des normes discursives par les locuteurs eux-mêmes. Dans le champ des études argumentatives, cette position est illustrée par exemple par Herman (2011: 51 et 59) lorsqu'il écrit que l'on ne peut " faire l'économie du rapport aux normes » car « c'est lorsque les acteurs thématisent dans leurs propos les règles du débat ou leur violation par l'adversaire qu'il a matière à analyse $»^{15}$. Dans le cas qui nous intéresse, la place accordée - ou refusée - aux procédés éristiques apparaît comme centrale.

Bien plus, les règles du raisonnement font elles-mêmes l'objet du dissensus, voire de l'attaque verbale : c'est souvent par une injure adressée à l'adversaire (le traitant de " fou », d'enfant, ou de "crétin») qu'un locuteur lui dénie la rationalité. Cette observation récurrente dans les larges corpus analysés par Angenot, se retrouve dans les polémiques entre intellectuels, prompts à se traiter de fous ou de charlatans (Angenot 2008: 23, 126-128, 262, 269-273; Oger 2003), et considérant par exemple que "l'idée que l'usage du mensonge ou de la ruse [...] est condamnable relève d'un postulat naïf ", Angenot y voit un appel à " historiciser la rationalité » (2008, resp. 87 et 423$)$ et à dessiner les contours des modalités particulières du raisonnement de chacun des grands systèmes argumentatifs qu'il a étudiés. On peut de la même manière, s'efforcer 
d'« historiciser » le recours à l'injure, pour repérer par exemple comment perdurent ou se transforment ces accusations de "folie » ou de «crétinerie » (Oger 2003), ou encore les assimilations animales : représentées dans les pamphlets, analysées également par Angenot (1982), elles dénient à l'adversaire l'appartenance à l'humanité, en même temps qu'elles produisent la dérision attachée à la caricature ${ }^{16}$.

11 L'existence de genres et de types de discours comme le pamphlet ou la satire, constituant un ensemble de traditions littéraires ou de pratiques médiatiques très attestées, offre en effet un cadre légitimant à l'usage de l'invective, qui se soutient ici d'exemples autorisés. Et si les catégories juridiques qui définissent l'injure et la diffamation ne sont guère opératoires pour l'analyse des corpus, les réflexions des linguistes, des sociologues, des politistes ou des juristes sur l'activité judiciaire et la jurisprudence nous renseignent sur les conditions dans lesquelles un discours dénoncé comme injurieux peut ou non bénéficier de l'excuse de l'humour, mentionnée plus haut, mais aussi des exceptions attachées aux genres satirique et pamphlétaire (Lecomte 2008 : 147-149 ; Vincent 2010, Lagorgette 2010, Passard 2009).

Le pamphlet retient particulièrement l'attention, en raison des relations étroites qu'il entretient avec le recours à l'injure. Les procédés, analysés par Angenot (1982), présentent de frappantes analogies avec les polémiques entre intellectuels, telles qu'elles se déploient, par tribunes interposées, dans les colonnes des grands quotidiens (Oger 2003). Mais au-delà du détail des procédés mobilisés, c'est bien le régime de « vitupération » (Hastings 2009) qui, centré sur la colère et l'indignation, y autorise les déplacements du discours de l'autre, et en définitive, l'ensemble des formes de l'agression, laissant dans certains cas le champ libre à la haine (Hastings $2009: 44)^{17}$. Micheli (2010) a montré comment le partage entre les émotions et les sentiments licites ou illicites dans l'argumentation ne peut être établi que dans un contexte donné et pour un groupe de locuteurs donné. Or les modalités de «l'argumentabilité » des émotions ainsi définie par Micheli, entrent en relation directe avec le recours à des procédés qui relèvent de la violence verbale, comme le montrent notamment les travaux cités sur le pamphlet et l'ethos pamphlétaire.

Lorsqu'il étudie les Grands récits, formations idéologiques majeures qui reposent, tout au long du $19^{e}$ siècle sur la lutte entre Révolution et Contre-Révolution, entre socialisme et antisocialisme, Angenot s'interroge aussi sur les formes de légitimation de la violence, qui a pu être considérée comme «le plus saint des devoirs » : arme légitime pour détruire une société fondée sur (et maintenue par) la violence, elle peut même dans le discours révolutionnaire se faire "violence nécessaire et purificatrice " alliée au « mépris » de la démocratie (Angenot 2003 : 221). Le culte de la force s'y associe à la revendication du discours de haine: «Soyez haineux, conservez farouche en votre cœur le fiel que vous cracherez plus tard au visage de tout ce qui est aujourd'hui en haut $»^{18}$.

14 Pour Angenot, le recours à la violence verbale se trouve partagé par toutes les grandes idéologies qui font l'objet d'une adhésion sans faille: dès lors qu'une «ligne » est rendue sacrée et fait l'objet de "dévotion », la rhétorique partisane est susceptible de mobiliser falsifications, ricanements, diffamations, bref "tous les moyens" pour "déshonorer les attaquants» (2008: 307). Ainsi donc se trouvent dessinées des situations de débat qui présentent les principales caractéristiques des polémiques: forte polarisation et visée de disqualification de l'adversaire (Amossy $2010: 26-27$ ). 

l'injure à la lumière des genres, mais aussi des positionnements qui tendent à construire - et parfois à stabiliser dans le temps - un "ethos de groupe » (Micheli 2010 : 354-361). De ce point de vue, c'est seulement dans le contexte historique précis d'une de ces arènes et dans le réseau serré d'un interdiscours spécifique que l'on pourra analyser le recours à l'injure comme le « discours de haine ».

\section{Historicisation de l'agression verbale : déplacements, circulations, transformations}

On trouvera dans les travaux d'historiens des analyses détaillées des contextes précis de l'émergence ou du déploiement de formes de l'agression verbale. Cette approche est illustrée par les nombreuses études de cas rassemblées par Bouchet et al. (2005), parfois analysées dans leurs formes discursives, toujours rattachées aux circonstances de leur apparition, aux caractéristiques de la situation sociale et politique, à celles de la trajectoire sociologique des locuteurs. Autre exemple, un chapitre consacré par Baecque au "grand spectacle de la transparence » montre comment, dès l'année 1789, se développe et s'institue un "espace de la transparence ", qui rompt avec la sphère publique des Lumières et l'appel à l'opinion éclairée, pour faire appel au peuple, légitimant la dénonciation publique des «traîtres ", «fuyards » et " comploteurs » : les adversaires y sont désignés à la vindicte, leurs noms accompagnés de descriptions ou qualificatifs injurieux, leurs tares physiques soulignées... (Baecque 1993 : 257-302). On ne s'étonnera certes pas que puisse être propice au déploiement de la violence verbale et de la pratique de l'injure un contexte révolutionnaire, comme de manière beaucoup plus générale toutes les situations qui voient s'intensifier les tensions politiques ${ }^{19}$. Plus simplement et plus couramment, l'agression verbale est associée aux situations de rivalité et de compétition politiques, comme les campagnes électorales.

Signalons également un type de contexte, plusieurs fois décrit dans des recherches fort différentes, et qui semble se prêter au déploiement de l'injure : la violence verbale la plus brutale s'y appuie sur l'indignation, pour ne pas dire la fureur, provoquée par une évolution politique, sociale ou morale jugée à la fois inacceptable et inéluctable, un bouleversement des valeurs qui serait imposé sans débat. Chanay analyse ainsi les lettres reçues par Noël Mamère - après le mariage homosexuel qu'il avait célébré à Bègles. Des lettres étonnamment semblables ont d'ailleurs été reçues, et en partie publiées, par Roselyne Bachelot (1999) après sa prise de position sur le PACS $^{20}$. Or cette configuration particulière, que Chanay qualifie d'« outrage réactionnaire » nous semble rejoindre celles qui sont décrites par des travaux aussi différents que les deux exemples qui suivent.

Pour revenir à la période révolutionnaire, Baecque écrit à propos des Actes des apôtres, publication satirique monarchiste, que cet organe polémique, qui se réclame de l'esprit aristocratique et de la tradition du bel esprit pour revendiquer le recours à des « saillies de gaieté cruelle » est «né d'une réaction défensive face à l'accélération du cours des événements révolutionnaires ", contre laquelle il ne semble plus y avoir d'autre recours (Baecque 1996). Beaucoup plus près de nous, Orkibi analyse la "rhétorique d'agitation » mise en œuvre en 2004 par les opposants au plan de désengagement de Gaza : exclus du débat public médiatisé, leur visée explicite de heurter et choquer se manifeste par le recours à l'obscénité (en l'espèce un parallèle entre l'évacuation d'habitants et la

Argumentation et Analyse du Discours, 8 | 2012 
déportation des juifs pendant la Shoah) qui peut être analysé comme un basculement dans la violence (2011: 156-157).

L'intérêt de se pencher sur ces descriptions détaillées d'arènes publiques spécifiques est aussi de repérer des filiations ou des généalogies d'une part, des transformations ou des discontinuités d'autre part. Ainsi l'injure sexuelle, telle qu'elle est dénoncée par les femmes politiques présente des traits communs avec celle qui visait Léon Blum (Vigreux 200521), dont le cas présente également l'intérêt de montrer comment l'affrontement politique peut prendre une forme spécifiquement haineuse. Quant aux duels politiques analysés par Baecque, ils soulignent comment les affrontements opposent (aussi) l'image des corps et - pourrait-on dire - des "tempéraments" opposés, faisant écho pour l'analyste du discours à d'autres catégories : l'ethos discursif en effet se soutient d'une image préalable (que parfois il retravaille) et dans la relation à l'injure, on a vu que l'ethos combatif occupait une place centrale (Amossy 1999).

Quant aux discontinuités ou ruptures, elles sont familières aux travaux diachroniques sur l'argumentation, qui montrent tout d'abord que les locuteurs ne disposent pas nécessairement dans une controverse de répertoires stables d'arguments (Chateauraynaud 2011), mais aussi, symétriquement, que la mobilisation à des dates différentes par des acteurs différents d'un même argument le soumet à des déplacements de sens (Rennes 2011). Or ces deux formes de circulations et de déplacements trouvent un écho dans les pratiques injurieuses : la première est illustrée par des cas où les locuteurs produisent une nouvelle configuration argumentative: c'est ainsi que la dénonciation de la violence verbale sexiste, associée à l'argument du genre se trouve constituée en ressource centrale en faveur de la parité (Oger 2011), ou que la "resignification subversive " du discours de haine parvient à en détourner le pouvoir destructeur (Butler 2004 : 225-244). Le second cas peut être illustré par un exemple emprunté à notre corpus d'ouvrages de femmes politiques : perçu comme une agression sexiste spécifiquement dirigée contre sa candidature, le barbouillage, par des excréments, de la permanence de Ségolène Royal pendant une campagne électorale trouve des précédents dans les "mais injurieux » repérés au 19e siècle dans le Quercy, qui appartiennent à des formes aujourd'hui largement oubliées de la violence rurale, et se trouvent réinvestis dans un contexte très différent (Royal 1996: 74 et Ploux 2005).

\section{Pacification et dépacification des débats publics}

21 La dernière forme de contextualisation que nous mentionnerons est celle de l'inscription dans des dispositifs médiatiques ou institutionnels spécifiques. S'agissant des premiers, nous nous permettrons de renvoyer, sans nous y attarder, le lecteur à des recherches qui se sont attachées au repérage de ces déplacements: le recul du pamphlet comme genre littéraire - signalé par Angenot (1982) qui situe l'âge d'or du pamphlet entre 1868 et $1968^{22}$ - n'empêche pas le déplacement vers d'autres lieux de l' ethos pamphlétaire, qui subit au passage quelques transformations: anonymat ou pseudonymat sur les forums de discussion, statut des locuteurs pour les tribunes publiées dans la presse (et à qui leur inscription sociale et politique interdit de parler, comme le pamphlétaire, «depuis un exil $»^{23}$ ), provocations autorisées ou orchestrées par des logiques marchandes (par exemple les « dérapages » - ou supposés tels - des Eric Zemmour ou des Dieudonné dans les émissions télévisées), émergence de nouveaux 
genres et de nouveaux locuteurs ${ }^{24}$ (Hastings et al. 2009 ; Amossy et Burger 2011 ; Amossy 2011).

Les dispositifs institutionnels nous semblent appeler une attention plus soutenue ici, dans la mesure où ils sont le lieu de paradoxes et de configurations plus complexes. La place de la conflictualité (et le cas échéant de la violence) dans l'argumentation a en effet été abordée à partir de recherches qui questionnent le fonctionnement de la démocratie, tant du point de vue de la théorie politique que de la description des arènes institutionnelles.

S'agissant de la confrontation des points de vue, la question des modalités de la délibération et/ou de la participation a alimenté une abondante production scientifique, dans les pays anglo-saxons puis en France. Ces réflexions, qu'elles soient plutôt normatives ou plutôt sociologiques, excluent d'emblée le recours à l'injure ou à la violence verbale comme telles: quels que soient les fondements et les représentations proposées de l'échange des points de vue, celui-ci exclue non seulement l'agression, mais aussi toute forme de coercition, ainsi que le marchandage, la promesse ou la menace, et même la négociation (Sintomer et Talpin 2011, Manin 2011). Un point pourtant retiendra notre attention, celui des grands modèles que l'on a pu dégager de ces recherches ${ }^{25}$.

$24 \mathrm{Au}$ modèle normatif habermassien, caractérisé notamment par un postulat de rationalité des participants, et une visée de consensus (Sintomer et Talpin 2011), un certain nombre de chercheurs ont apporté des prolongements ou des variantes qualifiées de "modèle conversationnel ». D'autres réflexions cependant ont alimenté une alternative, fondée sur la référence à la rhétorique cicéronienne et qualifiée de "modèle oratoire »: le principe ici défendu est celui d'une présentation systématique des points de vue opposés, par des participants qui prennent le rôle d'orateurs devant le reste de l'auditoire, et qui utilisent les ressources de la rhétorique et singulièrement «l'appel aux passions» (Manin 2011: 106; Urfalino 2005). On voit ici qu'à un échange entre participants, exclusivement fondé sur le logos, se trouve opposée une forme quasi-théâtralisée - et propice à l'expression des émotions - de la confrontation des points de vue.

C'est par ailleurs sur cette confrontation conflictuelle - mais non violente - des opinions que les partisans d'une "démocratie radicale» (Mouffe 2000, Cohen et Fung 2004), fondent l'idéal d'une démocratie "agonistique ", qui échapperait à la fois aux faux semblants d'une politique consensuelle, "sans adversaire" (Mouffe 2000: 108-128), et aux écueils de la violence. L'accent est mis ici sur la mise au jour du dissensus, mais le modèle oppose bien des "adversaires " et non des "ennemis » et derrière l'opposition proposée par Mouffe entre agonism et antagonism (2000 : 13), se lit celle, plus ancienne de l'agon et du polemos.

Pourtant lorsqu'il se penche sur les questions que pose ce modèle aux formes de la participation, Blondiaux ne manque pas, parmi les formes identifiables du conflit, de s'interroger sur la tolérance qu'il convient d'adopter à l'égard des «formes les moins policées d'expression politique ", des « discours qui ne respectent pas formellement les "règles du jeu" " ni les « formes autorisées » (Blondiaux 2008 : 134, et 2004).

Plus significative encore pour notre propos, l'opposition entre les deux " grammaires » de l'activité parlementaire définies par Heurtin (199926), et touchant quant à elles aux origines de la démocratie représentative en France : historiquement liées aux débats 
sur l'architecture qu'il convenait de donner à l'assemblée (la forme circulaire plaçant les orateurs dans des positions égales et symétriques pour la discussion, et face au président ; l'hémicycle induisant une confrontation entre l'auditoire et l'orateur placé à la tribune), la grammaire de la discussion et la grammaire critique s'opposent sur les modalités du débat parlementaire et sur la place de la rhétorique : ici aussi la place des passions est déterminante, car la grammaire critique commande un régime de parole caractérisé par le recours aux figures, à une diction ornée, à des gestes expressifs... qui ont pour but non pas seulement, comme dans la grammaire de la discussion, de convaincre, mais bien d'émouvoir, de frapper et même d'éblouir. Mieux encore, cette grammaire critique autorise même l'emportement, la passion, la véhémence, les injures...

Or l'Assemblée nationale, loin d'être le cadre exceptionnel de transgressions du règlement, apparaît par exemple comme un des hauts lieux de l'injure sexiste, et elle est décrite comme un temple de l'apostrophe ordurière et de l'agression sans préavis par Elisabeth Guigou, Ségolène Royal, Edith Cresson, Roselyne Bachelot, Muguette Jacquain, Simone Veil, Catherine Trautmann et bien d'autres. Certaines d'entre elles récusent d'ailleurs explicitement les artifices d'une éloquence parlementaire présentée comme emphatique, spectaculaire, agressive, en faisant le contre-modèle d'une parole politique féminine présentée comme plus naturelle, et de cet ethos féminin modéré dont nous avons parlé plus haut (Guigou 1997 : 160).

Quoiqu'il en soit de la question du genre et de l'entre-soi masculin des assemblées que sont venues troubler les femmes politiques ${ }^{27}$, le point qui nous intéresse ici concerne la manière dont, dans un dispositif institutionnel théâtralisé et marqué par l'usage d'une rhétorique "passionnée ", le passage à l'emportement, et par là à l'injure, se trouve rendu possible. Là encore la question a alimenté une production scientifique considérable et en dehors des ouvrages déjà cités ${ }^{28}$, nous pouvons citer le numéro de la revue Parlement(s) consacré en 2010 à la «violence des échanges en milieu parlementaire ». Les assemblées y apparaissent comme soumises à une dialectique de l'ordre et du désordre parlementaires qui en rend l'étude particulièrement intéressante pour étudier les formes licites du recours à l'injure en démocratie. D'un côté la délimitation d'un "unparliamentary language » (Ilie 2004) et la production de règlements sont censés entretenir la civilité des échanges (Baudot et Rozenberg 2010 : 7-8), de l'autre, des rixes, chahuts, mouvements collectifs, outrages, incivilités, accusations, "invective moqueuse» et insulte... (2010: 10-11), introduisent une violence pour une part ritualisée, «ni totalement jouée, ni totalement incontrôlée $»^{29}$.

D'autre part, ces travaux nous indiquent que l'opposition entre civilité et incivilité recoupe en bonne partie celle qui sépare working et talking parliaments : ici encore la dimension oratoire, publique et plus encore médiatisée (lors des séances de questions au gouvernement notamment) ne manque pas d'alimenter chahuts, apostrophes, injures... « La pacification serait ainsi du côté du huis-clos, de la collaboration transpartisane voire de l'expertise parlementaire, et la dé-pacification du côté du contrôle, de la publicité, de la tribune et du verbe » (Baudot et Rozenberg $2010: 16)^{30}$.

\section{Conclusion}

31 Nous chercherons pour conclure à résumer les conditions du recours licite à l'injure telles qu'elles apparaissent dans les travaux cités ici, et qui tiennent à la fois à un 
contexte d'intensification des tensions politiques (des révolutions ou des guerres aux campagnes électorales, en passant par le sentiment d'exclusion du débat et de frustration qui alimente l'« outrage réactionnaire ») mais aussi et surtout aux modèles de l'exercice de la parole qui sous-tendent les débats publics.

Tout à la fois ritualisées et souvent reçues par les victimes dans toute leur violence, les formes de l'injure se parent d'autant plus des excuses de l'humour, de la satire ou du chahut qu'elles apparaissent dans des arènes médiatisées et institutionnalisées. Et elles peuvent d'autant mieux trouver droit de cité en démocratie qu'elles peuvent s'appuyer à la fois sur le modèle dit " agonistique » (valorisant l'expression marquée du dissensus et la défense des opinions à travers un partage des rôles argumentatifs), en même temps que sur le modèle « oratoire » (valorisant l'usage des procédés rhétoriques, mais aussi l'expression des émotions ou des "passions»). Sans induire nécessairement la violence verbale, la conjonction de ces deux composantes facilite le passage vers les procédés éristiques: s'agissant des arènes institutionnelles, vers cette grammaire critique de la discussion dont les assemblées parlementaires offrent parfois des exemples spectaculaires et, s'agissant des arènes médiatiques, vers le déploiement de polémiques où fleurissent accusations ou insinuations croisées de racisme ou d'antisémitisme, de collaboration ou de folie.

Le continuum de l'argumentation polémique évoqué au début de cet article rejoint en cela le double mouvement qui affecte les assemblées parlementaires selon Baudot et Rozenberg: "les assemblées politiques modernes peuvent ainsi être situées sur un continuum opposant pacification et dé-pacification ». En ce sens si la théorie politique peut séparer clairement agon et polemos, l'analyse descriptive des formes et des lieux de la communication conflictuelle nous semble conduire davantage à l'hypothèse d'une continuité.

\section{BIBLIOGRAPHIE}

Amossy, Ruth \& Marcel Burger. 2011. «Introduction : la polémique médiatisée », Polémiques médiatiques et journalistiques. Le discours polémique en question(s) (dossier), Semen 31, 7-24

Amossy, Ruth. 2011. « La coexistence dans le dissensus. La polémique dans les forums de discussion ", Polémiques médiatiques et journalistiques. Le discours polémique en question(s) (dossier), Semen $31,25-42$

Amossy, Ruth (dir). 1999. Images de soi dans le discours ; la construction de l'ethos (Lausanne : Delachaux \& Niestlé)

Angenot, Marc. 1982. La parole pamphlétaire. Contribution à la typologie des discours modernes (Paris : Payot)

Angenot, Marc. 2003. « L'ennemi du peuple et l'agent de l'histoire (1800-1914) », Bonnafous, Simone, Pierre Chiron, Dominique Ducard \& Carlos Lévy, Argumentation et discours politique. Antiquité grecque et latine, Révolution française, Monde contemporain (Rennes : PUR), 213-224 
Angenot, Marc. 2008. Dialogue de sourds. Traité de rhétorique antilogique (Paris : Mille et une nuits)

Bachelot, Roselyne. 1999. Le Pacs entre haine et amour (Paris : Plon)

Baecque, Antoine de. 1996. «Les ridicules de l'homme nouveau. Un groupe de satiristes sous la Révolution ", Mots. Les langages du politique 48, 15-32

Baecque, Antoine de. 1993. Le corps de l'histoire. Métaphores et politique (Paris : Calmann-Lévy)

Baecque, Antoine de. 2007 [2002]. Les duels politiques. De Danton-Robespierre à Royal-Sarkozy (Paris : Hachette [Grasset])

Baudot, Pierre-Yves \& Olivier Rosenberg. 2010. « Introduction. Lasses d'Elias : des assemblées dépacifiées ?", Violence des échanges en milieu parlementaire (dossier), Parlement[s]. Revue d'histoire politique 14, 6-17

Beaumatin, Eric 2008. "Le trait "privé" / "public" en matière d'injure et de délits connexes : remarques linguistiques sur une distinction en droit français », Eric Desmons \& Marie-Anne Paveau (dir.), Outrages, insultes, blasphèmes : violences du langage et polices du discours (Paris : L'Harmattan), 71-90

Blondiaux, Loïc. 2004. «Prendre au sérieux l'idéal délibératif : un programme de recherche », Délibération et action publique (dossier), Revue suisse de science politique 10/4, 158-168

Blondiaux, Loïc. 2008. « Démocratie délibérative vs démocratie agonistique ? Le statut du conflit dans les théories et les pratiques de participation contemporaines ", Raisons politiques 30, 131-148 Bouchet, Thomas \& Jean Vigreux. 2010. «Violences parlementaires en perspective (1850-1900-1950-2000) », Violence des échanges en milieu parlementaire (dossier), Parlement[s]. Revue d'histoire politique $14,18-34$

Bouchet, Thomas, Matthew Legett, Jean Vigreux \& Geneviève Verdo (dir.). 2005. L'insulte (en) politique. Europe et Amérique latine du XIXe siècle à nos jours (Dijon : EUD)

Butler, Judith. 2004. Le pouvoir des mots. Politique du performatif, traduit de l'anglais par Charlotte Nordmann (Paris : Editions Amsterdam) [Excitable Speech, Routledge, 1997]

Chanay, Hugues de. 2008. « Outrages contre outrage : les lettres adressées à Noël Mamère à l'occasion du mariage de Bègles », Desmons, Eric \& Marie-Anne Paveau (dir.), Outrages, insultes, blasphèmes : violences du langage et polices du discours, (Paris : L'Harmattan), 151-176

Chateauraynaud, Francis. 2011. «Sociologie argumentative et dynamique des controverses : l'exemple de l'argument climatique dans la relance de l'énergie nucléaire en Europe ", L'argumentation au carrefour des disciplines : sciences du langage et sciences sociales (dossier), a contrario $16,131-150$

Cohen, Joshua \& Archon Fung. 2004. « Radical democracy », Délibération et action publique (dossier), Revue suisse de science politique 10/4, 169-179

Danblon, Emmanuelle. 2005. La fonction persuasive. Anthropologie du discours rhétorique. Origines et actualité (Paris : Colin)

Desmarchelier, Dominique. 2003. «Les mots de la violence, la violence des mots dans le discours politique français contemporain », Bonnafous, Simone, Pierre Chiron, Dominique Ducard \& Carlos Lévy, Argumentation et discours politique. Antiquité grecque et latine, Révolution française, Monde contemporain (Rennes : PUR), 225-234

Djordjevic, Ksenija. 2009. «La violence verbale après la violence de masse. Le procès de Slobodan Milošević devant le Tribunal pénal international », Mots. Les langages du politique 91, 115-122 
Djordjevic, Ksenija. 2010. «Violence verbale dans les conversations téléphoniques entre chefs de guerre en Bosnie : la parole au service de la violence de masse », Langage et société 132, 117-132

Dompner, Nathalie. 2010. «La légitimité politique en joue. Le chahut organisé des députés français sur la question des fraudes électorales depuis les années 1980 ", Violence des échanges en milieu parlementaire (dossier), Parlement [s]. Revue d'histoire politique 14, 35-48

Dury, Sandrine. 2003. Sept femmes au cœur de la tourmente (entretiens avec Laure Adler, Florence Arthaud, Lucie Aubrac, Roselyne Bachelot, Marie-Georges Buffet, Gisèle Halimi, Arlette Laguiller) (Paris : Editions Médicis)

Freud, Sigmund. 1992 [1905]. Le mot d'esprit et sa relation à l'inconscient (Paris : Gallimard)

Guigou, Elisabeth. 1997. Etre femme en politique (Paris : Plon)

Haddad, Galit. 2004. « La querelle du "défaitisme” en 1917 », Guerres et paix. Débats, combats, polémiques (dossier), Mots. Les langages du politique 76, 59-73

Hastings, Michel, Cédric Passard, Juliette Rennes. 2009. « Les mutations du pamphlet dans la France contemporaine ", Que devient le pamphlet ? (dossier), Mots. Les langages du politique 91, 5-17

Hastings, Michel. 2009. « De la vitupération. Le pamphlet et les régimes du “dire vrai” en politique ", Que devient le pamphlet? (dossier), Mots. Les langages du politique 91, 35-49

Herman, Thierry. 2011. « Le courant du Critical Thinking et l'évidence des normes : réflexions pour une analyse critique de l'argumentation ", L'argumentation au carrefour des disciplines : sciences du langage et sciences sociales (dossier), a contrario 16, 41-62

Heurtin, Jean-Philippe. 1999. L'espace public parlementaire : essai sur les raisons du législateur (Paris : PUF)

Ilie, Cornelia. 2004a. « Unparliamentary language: Insults as cognitive forms of confrontation » Dirven, R., R. Frank \& C. Ilie (eds.) Language and ideology, Vol. II: Descriptive cognitive approaches (Amsterdam : John Benjamins), 235-263

Ilie, Cornelia. 2004b. « Insulting as (un)parliamentary practice in the British and Swedish Parliaments: A rhetorical approach », Bayley, P. (ed.). Cross-Cultural Perspectives on Parliamentary Discourse (Amsterdam : John Benjamins), 45-86

Jonge, Emmanuel de \& Loïc Nicolas. 2009. «Limites et ambigüités rhétoriques du discours pamphlétaire. Vers l'abandon d'une pratique sociale? Que devient le pamphlet? (dossier), Mots. Les langages du politique 91, 51-65

Krieg-Planque, Alice (à paraître), « Une parole pamphlétaire : le "Petit glossaire de la guerre civile yougoslave" (1994) comme dictionnaire de combat », Cordell-Paris, Crystal (dir.), La rhétorique démocratique en temps de crise. Discours, délibération, légitimation, Actes du Colloque de Nice, 20-21 janvier 2011

Lagorgette, Dominique \& Pierre Larrivée. 2004. « Interprétation des insultes et relatiosn de solidarité », Langue française 141, 83-103

Lagorgette, Dominique. 2010a. « Présentation », in « Linguistique légale et demande sociale » (dossier), Langage et société 132, 5-14

Lagorgette, Dominique. 2010b. « "Le crime est dans l'œil de celui qui regarde le dessin" : l'analyse linguistique pour les tribunaux dans les procès Siné (2009) », Linguistique légale et demande sociale (dossier), Langage et société 132, 77-99

Largueche, Evelyne. 1983. L'effet injure. De la pragmatique à la psychanalyse (Paris : PUF) 
Largueche, Evelyne. 1993. L'injure à fleur de peau (Paris : L'Harmattan)

Largueche, Evelyne. 2011. « Et si l'injure ne se racontait pas ! », L'Homme 198-199, 355-366

Le Bart, Christian. 2009. « Le pamphlet politique aujourd'hui. Une catégorie honteuse ? Que devient le pamphlet? (dossier), Mots. Les langages du politique 91, 67-81

Lecomte, Didier. 2008. «Injures et outrages aux forces de police », Desmons, Eric \& Marie-Anne Paveau (dir.), Outrages, insultes, blasphèmes : violences du langage et polices du discours (Paris :

L'Harmattan), 137-150

Manin, Bernard. 2011. «Comment promouvoir la délibération démocratique ? Priorité du débat contradictoire sur la discussion », Démocratie délibérative (dossier), Raisons politiques 42, 83-113

Mercier, Arnaud (dir.). 2001. Dérision-contestation (dossier), Hermès 29

Micheli, Raphaël. 2010. L'émotion argumentée. L'abolition de la peine de mort dans le débat parlementaire français (Paris : Editions du Cerf)

Moïse, Claudine 2006. " Analyse de la violence verbale : quelques principes méthodologiques », Actes des XXVIe journées d'études sur la parole, Dinard, 103-114.

Mouffe, Chantal. 2000. The democratic paradox (London : Verso)

Oger, Claire. 2003. " "Crétins internationaux", "rancis de la guerre froide" et autres suicidés ; retour sur un corpus polémique ", Communication 22/2, 11-43

Oger, Claire. 2006. «Dialectique de la parole et du silence : émergence et fonction de l'injure sexiste en politique », Communication 25/1, 11-45

Oger, Claire. 2008a. «Du "parler cru" à l'insulte : niveaux de violence dans le discours sexiste en politique », Moïse, Claudine, Nathalie Auger, Béatrice Fracchiolla \& Christina Schultz-Romain (dir.), La violence verbale. Espaces politiques et médiatiques, vol. 1 (Paris : L'Harmattan), 47-61 Oger, Claire. 2008b. Le façonnage des élites de la République. Culture générale et haute fonction publique (Paris : Presses de Sciences Po)

Oger, Claire. 2011. « Argument du genre, ethos féminin et injure sexiste en politique. Analyse d'une configuration argumentative ", Duchêne, Alexandre \& Claudine Moïse (dir.), Langage, genre et sexualité (Paris : Editions Nota Bene), 91-113

Orkbi, Eithan. 2011. « Entre polémique et agitation : la violence verbale dans l'opposition au plan de désengagement de la bande de Gaza ", Polémiques médiatiques et journalistiques. Le discours polémique en question(s) (dossier), Semen 31, 145-162

Passard, Cédric. 2009. «Le pamphlet meurt-il de liberté ?», Que devient le pamphlet ? (dossier), Mots. Les langages du politique 91, 19-33

Paveau, Marie-Anne. 2008. «"J'irai cracher sur ta France”. Discours d'outrage aux emblèmes et symboles de l'Etat », Desmons, Eric \& Marie-Anne Paveau (dir.), Outrages, insultes, blasphèmes: violences du langage et polices $d u$ discours (Paris : L'Harmattan), 109-136

Plantin, Christian. 2003. « Des polémistes aux polémiqueurs », Declercq, Gilles, Michel Murat \& Jacqueline Dangel (dir.), La parole polémique (Paris : Champion), 377-498

Ploux, François. 2005. « Insultes au village (Haut-Quercy, XIXe siècle) », Bouchet, Thomas, Matthew Leggett, Jean Vigreux \& Geneviève Verdo (dir.), L'insulte (en) politique. Europe et Amérique latine du XIXe siècle à nos jours (Dijon : EUD) 
Rennes, Juliette. 2011. «Les formes de la contestation. Sociologie des mobilisations et théories de l'argumentation ", L'argumentation au carrefour des disciplines: sciences du langage et sciences sociales (dossier), a contrario 16, 151-173

Rosier, Laurence. 2006. Petit traité de l'insulte (Loverval : Labor)

Royal, Ségolène. 1997. La vérité d'une femme (Paris : Stock)

Sintomer, Yves \& Julien Talpin. 2011.« La démocratie délibérative face au défi du pouvoir », Démocratie délibérative (dossier), Raisons politiques 42, 5-13

Urfalino, Philippe. 2005. « La délibération n'est pas une conversation. Délibération, décision collective et négociation ", Délibération et négociation (dossier), Négociations 2 (Bruxelles : De Boeck), 99-114

Vigreux, Jean. 2005. « Travestir Léon Blum », Bouchet, Thomas, Matthew Leggett, Jean Vigreux \& Geneviève Verdo (dir.), L'insulte (en) politique. Europe et Amérique latine du XIXe siècle à nos jours (Dijon : EUD), 121-129

Viktorovitch, Clément. 2010. « Les commissions parlementaires à l'Assemblée nationale et au Sénat : un havre de paix ? » Violence des échanges en milieu parlementaire (dossier), Parlement [s]. Revue d'histoire politique 14, 90-110

Vincent, Diane. 2005. « Analyse conversationnelle, analyse du discours et interprétation des discours sociaux : le cas de la trash radio », Marges linguistiques 9, 165-175.

Vincent, Diane. 2010. « Mésinterprétation, plagiat, insulte et diffamation : objets de litige et matériaux de linguistes », Linguistique légale et demande sociale (dossier), Langage et société 132, $35-50$

Vincent, Diane, Olivier Turbide \& Marty Laforest. 2008. « Pour un modèle fonctionnel d'analyse du discours d'opposition », Moïse, Claudine,Nathalie Auger, Béatrice Fracchiolla \& Christina Schultz-Romain (dir.), La violence verbale. Espaces politiques et médiatiques, vol. 1 (Paris :

L'Harmattan), 81-108

\section{NOTES}

1. Alain Badiou, « Tout antisarkozyste est-il un chien? », Le Monde, 25 juillet 2008.

2. Nous ne pouvons reprendre ici le détail de l'analyse, et nous nous permettons de renvoyer sur ces points à Oger 2003 et Oger 2008a.

3. Il faut ajouter que nous nous intéressons à des formes de communication conflictuelle relatives aux "problèmes publics » et non aux interactions privées. L'opposition est nuancée par Amossy (2011: 37), mais Amossy et Burger (2011: 7) rappellent, à propos des polémiques publiques des enjeux qui concernent également notre problématique: «inscription dans un espace public» de discours «tributaires d'une dynamique et d'un jugement collectif à large échelle ».

4. Cette observation rejoint les travaux d'Amossy qui « voit dans l'argumentation un continuum allant de la co-construction des réponses à la confrontation violente des thèses antagonistes » (Amossy et Burger 2011 : 13), et de Plantin qui évoque différents degrés de polémicité (2003).

5. Par exemple la désignation "Tartarin des indices ", employée par François Mitterrand pour qualifier Raymond Barre. L'injure non-spécifique n'est en revanche pas adaptée aux traits particuliers de l'injurié, comme lorsqu'on le traite d'« andouille » (Larguèche 1983).

6. A la suite de R. Amossy, mais à la différence de C. Perelman et de M. Angenot, nous ne retenons pas la distinction entre argument ad hominem et argument ad personam. 
7. C'est une des raisons - parmi d'autres, que nous ne pouvons développer ici - pour lesquelles nous nous référons à l'injure et non à l'insulte, souvent définie comme sa composante linguistique.

8. Sur ces points, voir par exemple Lagorgette et Larrivée 2004 ; Rosier 2006.

9. Sur cette distinction, voir également Larguèche 1983, qui s'attache tout particulièrement à la place du tiers, dont la présence garantit la force et l'efficace de l'effet-injure. Ce point peut d'ailleurs être mis en relation avec l'opposition juridique entre contexte public et contexte privé, déterminante pour établir la gravité de l'injure (Beaumatin 2008, Larguèche 2011).

10. Sur l'excuse humoristique, voir les exemples commentés par Vincent (2005), Vincent et al. (2008: 107), et Passard (2009: 27-28).

11. On trouvera par exemple cette position dans Jonge et Nicolas (2009), ainsi qu'un point de vue, qui nous semble corrélatif, distinguant le registre pamphlétaire du registre polémique.

12. C'est le cas par exemple de Ségolène Royal ou d'Elisabeth Guigou. Roselyne Bachelot revendique au contraire un ethos combatif (Dury 2003 : 112). Pour plus de détails, voir Oger 2011.

13. Plus généralement d'ailleurs il nous semble possible d'avancer l'hypothèse que les normes discursives et plus précisément argumentatives, caractéristiques de tel ou tel milieu donné, s'organisent autour d'un modèle de gestion du rapport à l'autre dans le discours. Sur le lien entre cette "confrontation imaginaire » et la "discipline de corps" dans différents secteurs de la fonction publique, voir Oger $2008 \mathrm{~b}: 290-292$.

14. Pour une présentation des théories normatives de l'argumentation, voir notamment Danblon (2005: 97-113), qui en affine l'approche en introduisant la distinction entre «normes déontiques » et « normes régulatrices ».

15. Voir aussi Micheli (2010:25).

16. Voir la citation d'A. Badiou citée en tête de cet article, mais aussi, sur l'assimilation de la femme à une vache ou à une panthère, Oger 2008a.

17. Voir aussi les passages consacrés par Angenot à la « logique du ressentiment » (par exemple $2008: 343-350$ ).

18. Article du journal L'Egalité, cité par Angenot (2003:222).

19. L'intrication de la violence verbale de la violence physique a pu être analysée dans des contextes de conflits armés et la notion de brutalisation, proposée par l'historien G. L. Mosse et reprise par Haddad, pourrait fournir une catégorie heuristique pour en rendre compte (Haddad 2004 ; Djordjevic 2009 et 2010).

20. Forme d'union civile, instaurée par une loi de 1999 dont le vote a été précédé à l'Assemblée d'un débat particulièrement houleux et spectaculaire. R. Bachelot, seule députée de droite à avoir soutenu le texte, a été à ce titre violemment prise à partie, pendant et après le débat.

21. Voir aussi Baecque [2002] 2007 : 117-130 (sur le duel Thorez/Blum).

22. Sur le déclin, mais aussi la persistance du genre (ou de l'ethos associé), voir aussi Le Bart 2009 et Krieg-Planque (à paraître).

23. Nous empruntons l'expression à Danblon (2005: 53).

24. On pense aux rappeurs (Passard 2009 ; Lagorgette 2010) mais aussi à des figures plus isolées : Paveau évoque la manière dont un certain nombre de célébrités - aussi différentes que Serge Gainsbourg, Françoise Sagan, Jean Genet ou Béatrice Dalle - ont pu incarner en France, de manières très différentes, une "culture [...] de l'outrage", ou à défaut une relation de contestation ou de subversion aux normes et aux lois (Paveau 2008 : 127-128).

25. Sur ce point, Manin (2011) s'appuie notamment sur les travaux de Michael Schudson et de Gary Remer.

26. Voir notamment p. 129 le tableau qui résume l'opposition entre les deux " grammaires ».

27. Outre les témoignages des femmes politiques elles-mêmes, voir Baudot et Rozenberg (2010 :

16), qui citent les travaux incontournables de Mariette Sineau et Catherine Achin.

28. Ainsi que les travaux de Bouchet et al. (2005, 2010), Ilie (2004a, 2004b), Desmarchelier (2003). 
29. Sur la part ritualisée, voir Dompnier 2010, qui s'appuie sur l'opposition entre chahuts traditionnels et chahuts anomiques chez les lycéens.

30. L'observation ethnographique des commissions parlementaires menée par Viktorovitch, sans infirmer ce point de vue, le nuance toutefois. Par ailleurs, Royal (1996:69) donne un exemple d'apostrophe sexiste lancée lors de la formation d'une commission parlementaire (mais, il est vrai, pas au cours de ses travaux).

\section{RÉSUMÉS}

Cet article s'interroge sur les conditions de légitimation de la violence verbale et sur la description des arènes où elle est pratiquée comme une forme licite de l'adresse à autrui. Il s'appuie sur des recherches menées antérieurement, et qui ressortissent à deux types de corpus : les polémiques médiatisées d'une part, et d'autre part les ouvrages de femmes politiques qui dénoncent le recours à l'injure comme forme de la violence sexiste en politique. On rappelle la manière dont ces discours s'appuient sur des définitions antagonistes de l'ethos légitime dans le débat public : d'un côté le recours assumé à la conflictualité comme "règle du jeu politique ", de l'autre la condamnation de l'injure comme forme de violence symbolique. On propose ici des rapprochements avec des recherches menées dans plusieurs disciplines, et qui permettent d'éclairer les contextes dans lesquelles le recours à l'injure peut devenir licite, voire légitime. On insiste plus particulièrement sur un certain nombre de travaux portant sur les modèles qui soustendent la prise de parole dans le débat public - et sur la place que peuvent y occuper la conflictualité et la violence verbale.

This paper aims at questioning the conditions under which verbal violence can appear as legitimate, and the description that is made of public spheres where it can appear as acceptable discourse. Our work builds on previous research about two types of corpora: controversies that received a lot of media coverage on the one hand, and books written by female politicians on the other hand, books that denounce the recourse to insults as a specific form taken by sexist violence in politics. Two different conceptions of the legitimate ethos in public debate underlie these types of discourse: on the one hand the debaters accept the recourse to offensive language as part of the rules of the political game, and on the other hand, verbal abuse is condemned as a form of symbolic violence. These conceptions are here confronted to a set of interdisciplinary publications, which can shed light on contexts in which insult can become acceptable or even legitimate. This paper particularly puts the emphasis on research dealing with the different models that underlie proceedings in public debate, and the place given to conflict or verbal violence within each of them.

\section{INDEX}

Mots-clés : débat public, ethos légitime, injure, interdisciplinarité, violence verbale

Keywords : abuse, interdisciplinarity, legitimate ethos, public debate, verbal violence 
AUTEUR

CLAIRE OGER

Université Paris 13, LabSic EA 1807 